Estrutura de Áreas protegidas dos assentamentos de reforma agrária no município de Uberlândia-MG, Brasil: um estudo de ecologia de paisagem

Paula Guardenho Maywald, Oswaldo Marçal Júnior

\title{
ESTRUTURA DE ÁREAS PROTEGIDAS DOS ASSENTAMENTOS DE REFORMA AGRÁRIA NO MUNICÍPIO DE UBERLÂNDIA-MG, BRASIL: UM ESTUDO DE ECOLOGIA DE PAISAGEM
}

\section{Structure of protected areas of the agrarian reform settlements in the municipality of Uberlândia, state of Minas Gerais, Brazil: a landscape ecology study}

\author{
Paula Guardenho Maywald \\ Profa. Mestre, Centro Universitário do Triângulo Mineiro \\ paulaguardenho@yahoo.com.br
}

Oswaldo Marçal Júnior

Prof. Associado, Instituto de Biologia, UFU

marcaljr@ufu.br

Artigo recebido em 09/11/2011 e aceito para publicação em 13/12/2012

RESUMO: Este estudo avaliou a estrutura das áreas protegidas de assentamento de reforma agrária, no município de Uberlândia (MG). A pesquisa foi conduzida entre 2010 e 2011. Foram investigados 12 dos 15 assentamentos localizados em Uberlândia. Áreas de Proteção Permanente (APP) e Reservas Legais (RL) foram analisadas por meio de técnicas de mapeamento. Índices morfométricos foram calculados para oito das RL. A área total dos assentamentos foi de $12.756,87$ ha, dos quais 3.301,57 ha corresponderam a áreas de proteção (25,88\%). As RL, com 2.580,78 ha, representaram 20,23\% da área total e $78 \%$ das áreas protegidas. As APP somaram 720,79 ha (5,65\% da área total). Três assentamentos tiveram RL demarcadas em apenas um fragmento; nos outros, as RL dividiram-se de quatro a seis fragmentos. Com exceção de três assentamentos, todos apresentaram conectividade entre os fragmentos, inclusive com as APP. Zumbi dos Palmares apresentou maior efeito de borda nas áreas protegidas, enquanto os menores valores foram verificados em Nova Tangará. As áreas protegidas nos assentamentos rurais pesquisados equivalem a 167\% das áreas ocupadas pelos Parques e Reservas de Uberlândia.

Palavras-chave: Conservação, Ecologia, Agricultura, Assentamentos rurais, Unidades de Conservação.

ABSTRACT: This study evaluated the structure of the protected areas of agrarian reform settlements in the municipality of Uberlândia, state of Minas Gerais. The research was done between 2010 and 2011. We investigated 12 of the 15 settlements located in Uberlândia. Permanent protected areas (PPA) and Legal reserves (LR) were analysed by mapping techniques. Morphometric indexes were calculated for eight LR. The total area of the settlements was $12.756,87$ ha, of which $3.301,57$ ha were protected areas $(25.88 \%)$. The LR, with $2,580.78$ ha represented $20.23 \%$ of the total area and $78 \%$ of the protected areas. PPA added 720.79 ha (5.65\% of the total area). Three settlements had LR demarcated in just a fragment; in the other ones, LR became separated from four to six fragments. Except for three settlements, all of them presented connection among fragments, including with PPA. Zumbi of Palmares presented larger border effect in the protected areas, while the smallest values were verified in New Tangará. The protected areas in the researched rural settlements are equivalent to $167 \%$ of the occupied areas for Parks and Reserves of the municipality of Uberlândia.

Keywords: Conservation, Ecology, Agriculture, Rural settlement, Conservation Units. 
Estrutura de Áreas protegidas dos assentamentos de reforma agrária no município de Uberlândia-MG, Brasil:

um estudo de ecologia de paisagem

Paula Guardenho Maywald, Oswaldo Marçal Júnior

\section{INTRODUÇÃO}

A Reforma Agrária compreende o "conjunto de medidas para promover a melhor distribuição da terra, mediante modificações no regime de posse e uso, a fim de atender aos princípios de justiça social, desenvolvimento rural sustentável e aumento de produção" (INCRA, 2011). Essa concepção foi estabelecida pelo Estatuto da Terra (Lei 4.504, de 30/11/1964), que definiu a função social da propriedade a partir dos princípios de produtividade, trabalho, preservação ambiental e bem estar, como resposta à estrutura fundiária vigente no país. Assim, de 1979 a 1987, foram assentadas 105.778 famílias, em 7.247.245 ha, abrangendo 436 assentamentos. A partir da promulgação da Constituição de 1988, com a desconstituição do Estatuto da Terra, a reforma agrária deixou de ser realizada em bases territoriais e passou a ser feita pontualmente, por meio da implementação de projetos de assentamento, nas grandes propriedades improdutivas (SILVA, 2011). Com essa mudança, entre 1988 e 2006, foram implantados, em todo país, 7.230 Projetos de Assentamento de Reforma Agrária, com aproximadamente 800 mil famílias assentadas, em uma área de 57.305.522 ha (GIRARDI, 2011). Em 2008, a Mesorregião do Triângulo Mineiro/Alto Paranaíba apresentava 77 projetos de assentamentos de reforma agrária (106.395ha), conferindo à região, o primeiro lugar em número de ocupações no Estado, seguida das regiões do Norte de Minas e do Vale do Rio Doce (DATALUTA, 2011).

Os assentamentos de reforma agrária se constituem em unidades de produção agrícola que, independente do seu tamanho e estrutura, estão sujeitas à legislação ambiental brasileira, incluindo a exigência de licenciamento ambiental para o desenvolvimento de diversas atividades (MOURA et al, 2008). Essa legislação exige, para aprovação de cada projeto, a averbação de Reserva Legal (RL), além das áreas instituídas como Áreas de Proteção Permanente (APP) (BRASIL, 1965; 1981; 1989; 2001). A manutenção de áreas protegidas se mostra um fator essencial para garantir a conservação da biodiversidade (PRIMACK ; RODRIGUES, 2001).

O município de Uberlândia, região do Triângulo Mineiro, está inserido no domínio do Cerrado, bioma que sustenta uma extraordinária diversidade biológica (KLINK; MACHADO, 2005), mas que também suporta uma enorme pressão ambiental, sobretudo do setor agropecuário, motivos que determinam sua classificação como uma das áreas prioritárias para conservação, em todo o mundo (MYERS et. al., 2000). Atualmente, cerca de 2 milhões de $\mathrm{Km}^{2}$ originais do Cerrado foram transformados em pastagens plantadas, culturas anuais e outros tipos de uso. A destruição dos ecossistemas mantém-se acelerada, variando entre 22.000 a $30.000 \mathrm{Km}^{2}$ por ano. As áreas com vegetação nativa mais preservada se concentram na Serra do Espinhaço, centro-leste de Minas Gerais, Serra da Mesa, Goiás e norte do Distrito Federal e região da Ilha do Bananal, locais onde as características do terreno, tais como grande declividade, solos pouco profundos e sujeitos às inundações periódicas representam um entrave à implantação dos grandes projetos de agricultura (MACHADO et al., 2004). O Oeste da Bahia e o Sul dos Estados do Piauí e do Maranhão ainda possuem áreas em bom estado de conservação. No entanto, por representarem a "última fronteira agrícola", observa-se uma rápida expansão do agronegócio na região (TRINDADE et al., 2010, ANDRADE; GOMES, 2012). O Triângulo Mineiro sofre fortes impactos da expansão agropecuária, das atividades mineração e do crescimento urbano. De fato, é considerada a região mais degradada do Estado (FERNANDES et. al., 2005).

Diversos trabalhos têm procurado relacionar a reforma agrária com o meio ambiente, no Brasil (BATISTELLA; CASTRO, 2001; BEDUSCHI FILHO 2003; MARGARIDO, 2004; RODRIGUES et al, 2004; BRANDÃO; SOUZA JR., 2006; PEREIRA, 2007; BASTOS e BRASIL, 2008; SOARES, 2008). Mas a despeito de 114.688,86ha terem sido destinados à reforma agrária no Triângulo Mineiro/Alto Paranaíba, no período 1986-2005 (DATALUTA, 2011), nenhum estudo foi desenvolvido na região, avaliando essa temática, do ponto de vista ecológico. A presente pesquisa foi desenvolvida com intuito de contribuir para o preenchimento dessa lacuna, a partir de uma análise integrada dos aspectos físicos e ecológicos e, em última análise, de sua interação com fatores sócio econômicos. Nessa perspectiva, buscou-se referencial na Ecologia da Paisagem, área do conhecimento que

Soc. \& Nat., Uberlândia, 25 (1): 75-90, jan/abr/2013 
oferece tanto bases teóricas como ferramentas metodológicas para uma análise ambiental integrada (METZGER, 2001). Esta abordagem tem como ponto central o reconhecimento da dependência espacial entre as unidades da paisagem; ou seja, de que o funcionamento dessas unidades depende das interações mantidas com a sua vizinhança, com ênfase na importância do contexto espacial sobre os processos ecológicos e no caráter fundamental das interações. Assim, o presente estudo teve como objetivo avaliar a estrutura das áreas de proteção de projetos de assentamentos de Reforma Agrária, no município de Uberlândia (MG).

\section{MATERIAL E MÉTODOS}

O município de Uberlândia, MG - 1856'38"' de Latitude Sul e $48^{\circ} 18^{\prime} 39^{\prime \prime}$ de Longitude Oeste, na região do Triângulo Mineiro, extremo Oeste do Estado de Minas Gerais. O município tem área de 4.115,09 $\mathrm{km}^{2}$, dos quais $3.896,09 \mathrm{~km}^{2}$ correspondem à área rural e $219 \mathrm{~km}^{2}$ à área urbana. Atualmente, 77.608ha do município se constituem em áreas com vegetação natural (campo cerrado, cerrado, matas ciliares, veredas e brejos), com destaque para veredas e brejos (27.212ha) e matas ciliares (18.212ha). A maior parte do solo, no entanto, é composta por áreas de pastagens (211.275ha), que correspondem a 51,36\% da sua área total, seguida por áreas de agricultura (70.553ha; $17,44 \%$ ) e de reflorestamento (16.719ha; 4,06\%) (PMU, 2010).

A pesquisa foi conduzida no período de 2010 a 2011, a partir de uma abordagem ecológica, baseada nos princípios da Ecologia de Paisagem (METZGER, 2001). O conceito de métricas da paisagem, adaptado de Forman e Godron (1986), foi aplicado às Reservas Legais, aqui tomadas como fragmentos de vegetação, de modo a permitir a quantificação da composição e configuração dessas paisagens.

Foram investigados 12 dos 15 assentamentos de reforma agrária localizados no município de Uberlândia, a saber: Rio das Pedras, Nova Palma, Palma da Babilônia, Zumbi dos Palmares, Nova Tangará, Flávia Nunes, Canudos, Emiliano Zapata, Paciência, José dos Anjos, Florestan Fernandes e Eldorado dos Carajás. Não foram pesquisados os assentamentos Maringá-Monte Castelo e Valci dos Santos, por se estendem para além do município de Uberlândia, e o Projeto de Assentamento na Fazenda Douradinho, em função da fase inicial de implantação (Figura 1; Quadro 1).

As Reservas Legais (RL) e às Áreas de Proteção permanente (APP) foram analisadas por meio de técnicas de mapeamento, sendo consideradas as seguintes variáveis: área total de conservação, áreas das APP e RL, número de fragmentos das RL, status das RL quanto ao isolamento e conectividade com as demais áreas de conservação do assentamento. Utilizou-se o programa AutoCAD 2006, versão em português, da Autodesk, no tratamento e análise dos dados físicos dessas áreas. A partir dos desenhos vetoriais no formato .dwg, foram delimitadas as APP dos assentamentos e estabelecidos os valores de área total $\left(\mathrm{m}^{2}\right)$ e de perímetro (metro linear). Para as RL, utilizando-se a ferramenta de cópias paralelas, foram estabelecidas as áreas de efeito de borda. A largura de borda foi definida em 30m, de acordo com Lima-Ribeiro (2008). 
Estrutura de Áreas protegidas dos assentamentos de reforma agrária no município de Uberlândia-MG, Brasil: um estudo de ecologia de paisagem

Paula Guardenho Maywald, Oswaldo Marçal Júnior

Figura 1. Distribuição dos Projetos de Assentamento de Reforma Agrária no município de Uberlândia, MG.

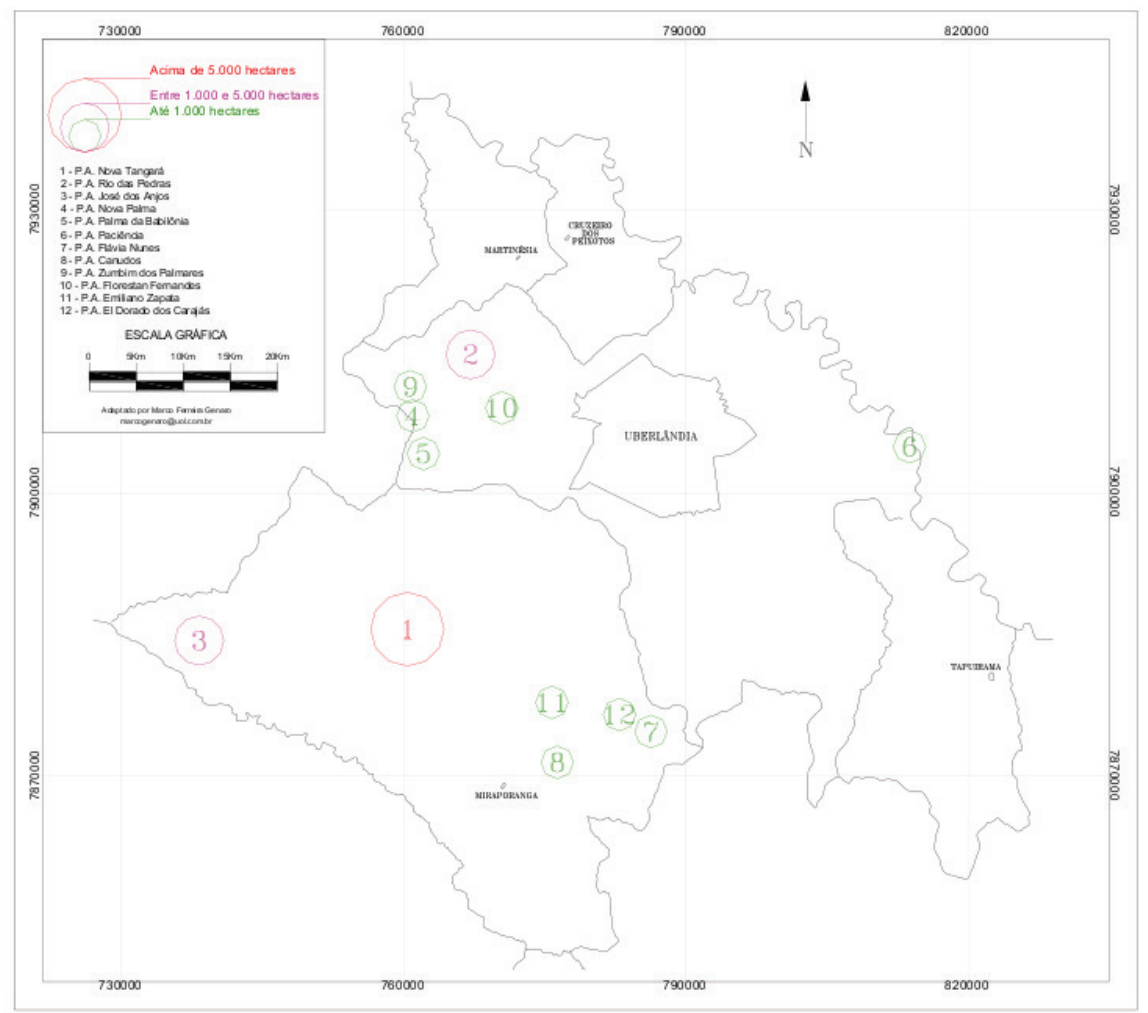

Fonte: Gontijo \& Batista (2009).

Quadro 1. Informações gerais dos Projetos de Assentamentos de Reforma Agrária em Uberlândia, MG

\begin{tabular}{|c|c|c|c|c|c|c|c|}
\hline & & & & \multicolumn{3}{|c|}{ Área (ha) } \\
Assentamento & Ano & \multirow{2}{*}{$\begin{array}{c}\text { Total } \\
\text { de criaçáo }\end{array}$} & $\begin{array}{c}\text { de famí- } \\
\text { lias }\end{array}$ & $\begin{array}{c}\text { Base cartográ- } \\
\text { fica utilizada }\end{array}$ & $\begin{array}{c}\text { Registrada } \\
\text { (Matrícula) }\end{array}$ & $\begin{array}{c}\text { Levantada } \\
\text { (Vistoria) }\end{array}$ & $\begin{array}{c}\text { Mapa*** } \\
\text { Perímetro } \\
(\mathrm{m}) * * *\end{array}$ \\
\hline Rio das Pedras & 1998 & 87 & INCRA & 1908,99 & ND & - & - \\
\hline Nova Palma & 1999 & 15 & INCRA & 455,92 & 459,15 & 445,64 & 12159,6 \\
\hline Palma da Babilônia & 1999 & 13 & INCRA & 458,32 & 457,14 & 465,67 & 12178,23 \\
\hline Zumbi dos Palmares & 1999 & 22 & INCRA & 492,04 & 547,65 & 546,37 & 12806,77 \\
\hline Nova Tangarán & 2003 & 250 & INCRA & 5097,61 & 5090,03 & 5096,03 & 35636,23 \\
\hline Flávia Nunes & 2004 & 17 & INCRA & 433,71 & 426,93 & 414,83 & 9350,45 \\
\hline Canudos & 2004 & 24 & INCRA & 585,74 & 682,63 & 667,63 & 16589,56 \\
\hline *Emiliano Zapata & 2004 & 24 & INCRA & 645,22 & ND & 638,02 & 12075,89 \\
\hline Paciência & 2004 & 25 & PDA & 468,94 & ND & - & - \\
\hline *José dos Anjos & 2005 & 45 & INCRA & 1015,91 & ND & 1013,40 & 17620,99 \\
\hline *Florestan Fernandes & 2005 & 22 & INCRA & $532,4 * *$ & $494,45 * *$ & 494,45 & 10466,17 \\
\hline *Eldorado dos Carajás & 2006 & 30 & INCRA & 568,44 & 608,80 & 596,90 & 10321,82 \\
\hline
\end{tabular}

$\mathrm{ND}=$ Laudo não disponível; *Não apresenta mapa digitalizado das RLs. ** Somente parte da fazenda foi destinada ao assentamento. *** De acordo com dados do INCRA. Org. dos autores. 
As bases cartográficas dos assentamentos foram obtidas junto ao INCRA. Para Paciência foi utilizado como fonte o mapa do Plano de Desenvolvimento Ambiental do assentamento. A conectividade entre as áreas de proteção não pode ser avaliada para os assentamentos Emílio Zapata, Florestan Fernandes e Eldorado do Carajás, pela falta de RL digitalizada.

Os índices morfométricos foram calculados para as RL dos oito assentamentos que apresentaram dados disponíveis. As expressões algébricas para esses cálculos foram obtidas pelo programa de domínio público, FRAGSTATS (versão 2,0) (MCGARIGAL e MARKS, 1994). Os índices calculados foram:

- Área (A): expressa a área total $\left(\mathrm{m}^{2}\right)$ de cada fragmento, dividido por 10.000 (conversão para hectare).

- Perímetro (P): é a medida linear externa do contorno de cada fragmento, incluindo o de clareiras internas, quando existentes.

- Índice de forma (IF): é igual ao perímetro (m) do fragmento dividido pela raiz quadrada de sua área $\left(\mathrm{m}^{2}\right)$. Os valores são maiores ou iguais a um, sem limites ou unidade, sendo que quanto mais próximos de um, mais circular é o fragmento.

- Área interna (AI): definida como a área $\left(\mathrm{m}^{2}\right)$ do fragmento que não é afetada por uma certa faixa de vegetação ou borda (efeito de borda), dividido por 10.000 (conversão para hectare). Valores sempre maiores do que zero e sem limite.

- Efeito de borda (EB): definido pela diferença entre a área de um fragmento e a área interna deste fragmento.

- Número de áreas internas (NAI): definida como o número de áreas internas de um fragmento que não sofrem efeito de borda. Quanto maior o número de áreas internas, para fragmentos de mesma área, mais irregular será a sua forma.

- Índice de área interna (IAI): definida como o percentual do fragmento encontrado no interior (excetuando-se a área de borda). Quanto mais próximo de zero é este índice, menor é o percentual em relação à área do fragmento.

\section{RESULTADOS}

Os assentamentos rurais estudados totalizaram uma área de $12.756,87$ ha, dos quais $3.301,57$ ha constituíram-se em áreas de proteção $(25,88 \%)$. Essas áreas variaram de 97,35ha (Nova Palma) até 1.340,47ha (Nova Tangará), com uma média de 275,13ha/assentamento. Canudos apresentou a maior proporção de áreas protegidas $(44,76 \%$ da área total do assentamento), enquanto Florestan Fernandes e Eldorado dos Carajás mostraram as menores (20\%) (Tabela 1).

As RL totalizaram 2.580,78 ha, representando $20,23 \%$ da área total dos assentamentos e $78 \%$ das áreas protegidas. A maior RL foi registrada em Nova Tangara (1010,83 ha) e a menor em Flávia Nunes (84,03 ha). O assentamento Paciência apresentou o maior percentual de RL (23,77\% da área do assentamento), enquanto que Rio das Pedras e Nova Tangará mostraram os menores valores $(19,77$ e $19,84 \%$, respectivamente) (Tabela 1).

As APP somaram 720,79 ha $(5,65 \%$ da área total). A maior APP foi registrada no assentamento Nova Tangará $(329,64$ ha) e a menor em Nova Palma $(7,47)$. Em termos proporcionais, as maiores e menores áreas de APP foram registradas nos assentamentos Canudos e Rio das Pedras (23,86 e 0,7\%, respectivamente). Florestan Fernandes e Eldorado dos Carajás não apresentaram dados para cálculo de área das APP (Tabela 1). 
Estrutura de Áreas protegidas dos assentamentos de reforma agrária no município de Uberlândia-MG, Brasil: um estudo de ecologia de paisagem

Paula Guardenho Maywald, Oswaldo Marçal Júnior

Tabela 1. Áreas de proteção de assentamentos de Reforma Agrária, no município de Uberlândia, MG.

\begin{tabular}{|c|c|c|c|c|c|c|c|}
\hline \multirow[t]{2}{*}{ Nome } & \multirow[t]{2}{*}{$\begin{array}{l}\text { Área do assenta- } \\
\text { mento (ha) }\end{array}$} & \multicolumn{2}{|c|}{$\begin{array}{c}\text { Reserva Legal } \\
\text { (RL) }\end{array}$} & \multicolumn{2}{|c|}{$\begin{array}{c}\text { Área de Proteção } \\
\text { Permanente } \\
\text { (APP) }\end{array}$} & \multicolumn{2}{|c|}{ Total de áreas protegidas } \\
\hline & & ha & $\%$ & ha & $\%$ & ha & $\%$ \\
\hline Rio das Pedras & 1908,99 & 377,44 & 19,77 & 13,39 & 0,7 & 390,83 & 20,47 \\
\hline Nova Palma & 445,64 & 89,88 & 20,17 & 7,47 & 1,68 & 97,35 & 21,85 \\
\hline Palma da Babilônia & 465,67 & 106,13 & 22,79 & 8,11 & 1,74 & 114,24 & 24,53 \\
\hline Zumbi dos Palmares & 546,37 & 112,4 & 20,57 & 17,25 & 3,16 & 129,65 & 23,73 \\
\hline Nova Tangará & 5096,03 & 1010,83 & 19,84 & 329,64 & 6,47 & 1340,47 & 26,3 \\
\hline Flávia Nunes & 414,83 & 84,03 & 20,26 & 35,22 & 8,49 & 119,25 & 28,75 \\
\hline Canudos & 667,63 & 139,54 & 20,9 & 159,31 & 23,86 & 298,85 & 44,76 \\
\hline Emiliano Zapata ${ }^{1}$ & 638,02 & 127,6 & 20 & 77,98 & 12,22 & 205,58 & 32,22 \\
\hline Paciência & 468,94 & 111,48 & 23,77 & 27,8 & 5,93 & 139,28 & 29,7 \\
\hline José dos Anjos ${ }^{3}$ & 1013,40 & 203,18 & 20,05 & 44,62 & 4,4 & 247,8 & 24,45 \\
\hline Florestan Fernandes ${ }^{2}$ & 494,45 & 98,89 & 20 & - & - & 98,89 & 20 \\
\hline Eldorado dos Carajás ${ }^{2}$ & 596,90 & 119,38 & 20 & - & - & 119,38 & 20 \\
\hline TOTAL & 12756,87 & 2580,78 & 20,23 & 720,79 & 5,65 & 3301,57 & 25,88 \\
\hline
\end{tabular}

1- Não apresenta mapa digitalizado da RL; 2- Não apresenta mapa digitalizado da AP, sendo consideradas as áreas mínimas legal para a RL e AP; 3 - Dados sobre a Reserva Legal retirados do Plano de Desenvolvimento Ambiental (PDA).

Org. dos autores.

Três assentamentos apresentaram RL demarcadas em apenas uma área/fragmento (Nova Palma, Flávia Nunes e José dos Anjos). Em Nova Tangará, a RL foi dividida em três fragmentos. Nas demais, as RL foram separadas em quatro (Zumbi dos Palmares); cinco (Rio das Pedras, Palma da Babilônia e Paciência) ou seis (Canudos) fragmentos (Tabela 2).

Foi observada conectividade entre os fragmentos, em maior (Rio das Pedras e Canudos) ou menor grau (Zumbi dos Palmares e Paciência). Todos os assentamentos, com dados disponíveis, apresentaram fragmentos conectados com as APP. Destaque para o assentamento Rio das Pedras, onde os cinco fragmentos se mostraram conectados por intermédio da APP (Tabela 2).

Os resultados referentes aos índices morfométricos são apresentados na Tabela 3 .

As menores áreas de fragmentos (Fi) foram demarcadas nos assentamentos Palma da Babilônia, Zumbi dos Palmares e Canudos. O maior fragmento (992,98ha) foi encontrado em Nova Tangará (F2). A média dos fragmentos foi de 74ha.

Os menores valores de índices de forma foram registrados para os fragmentos $\mathrm{F} 1$, em Paciência
$(1,00) ; \mathrm{F} 5$ em Canudos (1,06); F3 e F4, no Rio das Pedras (1,10); F1, F3 e F2, na Palma da Babilônia (1,12; 1,14 e 1,16; respectivamente); e F1 em Nova Palma $(1,18)$. Os maiores índices foram registrados no F2 de Nova Tangará $(4,08)$ e F1 de Zumbi dos Palmares $(3,07)$. 
Estrutura de Áreas protegidas dos assentamentos de reforma agrária no município de Uberlândia-MG, Brasil: um estudo de ecologia de paisagem

Paula Guardenho Maywald, Oswaldo Marçal Júnior

Tabela 2. Número fragmentos das Reservas Legais (RL) e seu nível de conectividade, nos de assentamentos de Reforma Agrária do município de Uberlândia, MG.

\begin{tabular}{|c|c|c|}
\hline Assentamento & Número de fragmentos & Conectividade \\
\hline Rio das Pedras & 5 & Todos conectados entre si por meio de APP \\
\hline Nova Palma & 1 & Conectado com APP \\
\hline Palma da Babilônia & 5 & $\begin{array}{l}\text { F1 - conectado com APP e isolado dos demais; F2, F3 e F5 - conectados } \\
\text { por meio de APP; F4 e F5 são separados por estrada municipal }\end{array}$ \\
\hline Zumbi dos Palmares & 4 & Fragmento grande conetado à APP e os outros três, pequenos e isolados \\
\hline Nova Tangará & 3 & $\begin{array}{l}\mathrm{F} 1 \text { - conectado à APP e isolado dos demais; F2 e F3 - conectados entre si } \\
\text { por meio de APP }\end{array}$ \\
\hline Flávia Nunes & 1 & Conectado com APP \\
\hline Canudos & 6 & Todos conectados entre si por meio de APP \\
\hline Emiliano Zapata & RL não digitalizada & - \\
\hline Paciência & 5 & $\mathrm{~F} 1, \mathrm{~F} 2$ e F4 conectados à APP mas isolados entre si; F3 e 5 isolados \\
\hline José dos Anjos & $1^{*}$ & Conectado com APP \\
\hline Florestan Fernandes & RL não digitalizada & - \\
\hline Eldorado dos Carajás & RL não digitalizada & - \\
\hline
\end{tabular}

A maioria das áreas de RL apresentou um único núcleo de área interna, sendo que F2 e F3 de Zumbi dos Palmares tiveram valor zero e dois núcleos, respectivamente. Os menores valores de área interna foram registrados em fragmentos dos assentamentos de Zumbi dos Palmares, com 0,55ha e 2,07ha (21,37 e $27,53 \%$ de índices de área interna, respectivamente); e de Canudos com 1,23ha(30,32\%) e 1,83ha (34,64\%). Nova Tangará apresentou as maiores áreas demarcadas para RL, com 913,30ha (F2) e 120,86ha (F1). Estas áreas apresentaram índices de área interna de 91,98 e $88,49 \%$; respectivamente.

Os resultados de IAI para os fragmentos pesquisados são apresentados na Figura 2. Onze fragmentos tiveram IAI superiores a $80 \%$, incluindo quatro dos cinco fragmentos de Rio das Pedras (RIOF2, RIOF3, RIOF4 E RIOF5). Os fragmentos menos afetados pelo Efeito de borda foram registrados em Nova Tangará, onde TANF2 e TANF1 apresentaram IAI de 91,98\% e $88,49 \%$, respectivamente (correspondendo a áreas afetadas pelo efeito de borda de $8,02 \%$ e $11,51 \%$, respectivamente). Os fragmentos mais afetados pelo efeito de borda foram encontrados nos assentamentos de Canudos (CANF2, CANF1, CANF6) e Zumbi dos Palmares (ZUMF2, ZUMF4, ZUMF3). Esse último registrou os maiores efeitos de borda, sendo que o fragmento ZUMF2 teve $100 \%$ da sua área $(1,29 \mathrm{ha})$ afetada, enquanto os fragmentos ZUMF4 e ZUMF3 tiveram 1,38 ha $(78,63 \%)$ e 1,99ha $(72,47 \%)$ de área afetada, respectivamente. 
Estrutura de Áreas protegidas dos assentamentos de reforma agrária no município de Uberlândia-MG, Brasil: um estudo de ecologia de paisagem

Paula Guardenho Maywald, Oswaldo Marçal Júnior

Tabela 3. Índices morfométricos das Reservas Legais em assentamentos de reforma agrária do município de Uberlândia, MG

\begin{tabular}{|c|c|c|c|c|c|c|c|c|}
\hline \multirow{2}{*}{ Assentamentos } & \multicolumn{8}{|c|}{ Indices morfométricos } \\
\hline & $\mathrm{RL}$ & $\mathrm{A}$ & $\mathrm{P}$ & IF & $\mathrm{AI}$ & EB & NAI & IAI \\
\hline \multirow{5}{*}{ Rio das Pedras } & F1 & 66,24 & 4455 & 1,37 & 52,58 & 13,66 & 1 & 79,38 \\
\hline & F2 & 116,76 & 7037 & 1,63 & 96,21 & 20,55 & 1 & 82,40 \\
\hline & F3 & 62,45 & 3471 & 1,10 & 52,45 & 10 & 1 & 83,98 \\
\hline & F4 & 48,85 & 3079 & 1,10 & 40,06 & 8,8 & 1 & 81,99 \\
\hline & F5 & 83,14 & 5262 & 1,44 & 67,79 & 15,34 & 1 & 81,55 \\
\hline \multirow[t]{2}{*}{ Nova Palma } & $\mathrm{F} 1$ & 89,05 & 4464 & 1,18 & 76,04 & 13,02 & 1 & 85,38 \\
\hline & F1 & 43,67 & 2957 & 1,12 & 35,21 & 8,47 & 1 & 80,61 \\
\hline \multirow{4}{*}{ Palma da Babilônia } & $\mathrm{F} 2$ & 5,46 & 1080 & 1,16 & 2,65 & 2,8 & 1 & 48,70 \\
\hline & F3 & 10,27 & 1456 & 1,14 & 6,29 & 3,98 & 1 & 61,23 \\
\hline & F4 & 16,75 & 2116 & 1,29 & 10,86 & 5,88 & 1 & 64,87 \\
\hline & F5 & 19,35 & 2620 & 1,49 & 11,96 & 7,39 & 1 & 61,81 \\
\hline \multirow{4}{*}{ Zumbi dos Palmares } & F1 & 145,84 & 14841 & 3,07 & 118,69 & 27,15 & 1 & 81,38 \\
\hline & F2 & 1,29 & 589 & 1,29 & 0 & 1,29 & 0 & 0 \\
\hline & F3 & 7,5 & 2184 & 1,99 & 2,07 & 5,44 & 2 & 27,53 \\
\hline & F4 & 2,59 & 888 & 1,38 & 0,55 & 2,03 & 1 & 21,37 \\
\hline \multirow{3}{*}{ Nova Tangará } & F1 & 136,58 & 5393 & 1,15 & 120,86 & 15,72 & 1 & 88,49 \\
\hline & F2 & 992,98 & 51377 & 4,08 & 913,30 & 79,68 & 1 & 91,98 \\
\hline & F3 & 61,25 & 5176 & 1,65 & 46,38 & 14,88 & 1 & 75,71 \\
\hline Flávia Nunes & F1 & 83,92 & 48,97 & 1,34 & 69,66 & 14,26 & 1 & 83,00 \\
\hline \multirow{6}{*}{ Canudos } & F1 & 5,29 & 1385 & 1,51 & 1,83 & 3,46 & 1 & 34,64 \\
\hline & F2 & 4,06 & 1143 & 1,42 & 1,23 & 2,83 & 1 & 30,32 \\
\hline & F3 & 41,42 & 3274 & 1,27 & 32,16 & 9,26 & 1 & 77,65 \\
\hline & F4 & 49,95 & 4491 & 1,59 & 36,84 & 13,1 & 1 & 73,76 \\
\hline & F5 & 19,38 & 18,6 & 1,06 & 14,28 & 5,09 & 1 & 73,72 \\
\hline & F6 & 6,37 & 1252 & 1,24 & 3,18 & 3,19 & 1 & 49,91 \\
\hline \multirow{5}{*}{ Paciência } & F1 & 17,12 & 1658 & 1 & 12,48 & 4,64 & 1 & 72,9 \\
\hline & F2 & 3,19 & 825 & 1,15 & 1,07 & 2,12 & 1 & 33,69 \\
\hline & F3 & 20,54 & 1963 & 1,08 & 15 & 5,54 & 1 & 73,03 \\
\hline & F4 & 56,45 & 3204 & 1,07 & 47,16 & 9,29 & 1 & 83,55 \\
\hline & F5 & 15,72 & 2027 & 1,28 & 10,2 & 5,52 & 1 & 64,88 \\
\hline Total & 30. & 2233,43 & - & - & 1898,04 & 334,38 & - & 84,98 \\
\hline
\end{tabular}

RL - Reserva legal; Fi - Fragmento; A - Área (ha); P- Perímetro (m); IF - Índice de Forma; AI - Área interna (ha); EB - Efeito de borda (ha); NAI - Número de áreas internas; IAI - Índice de área interna.

Org. dos autores.

Figura 2. Valores (\%) ordenados dos Índices de Área Interna (IAI) dos fragmentos constituintes das Reservas Legais dos assentamentos rurais pesquisados em Uberlândia, MG

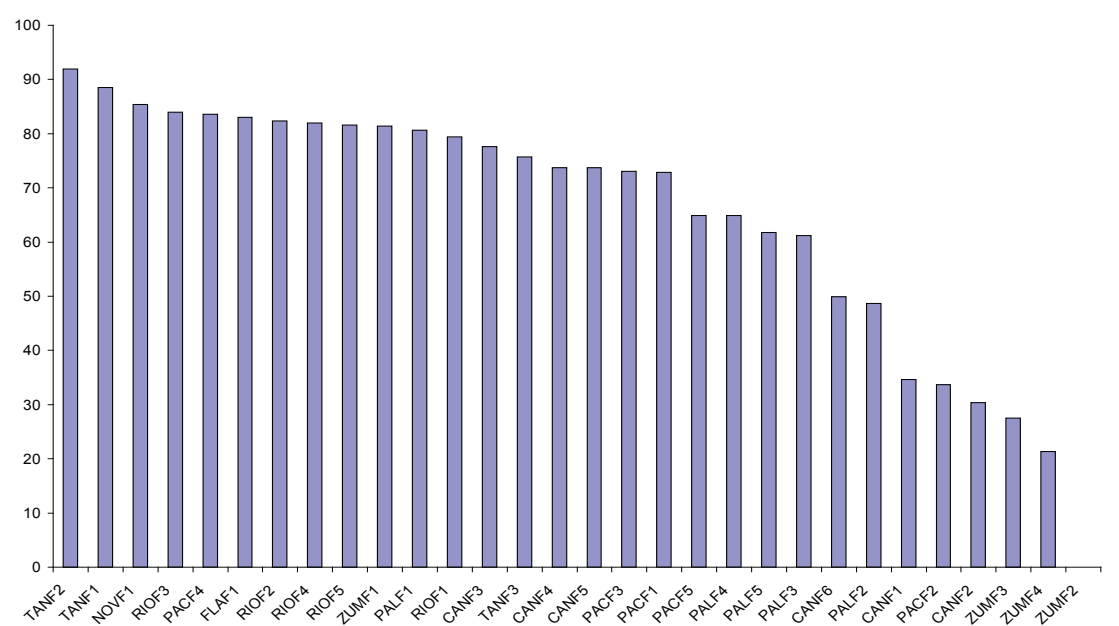

Org. dos autores. 


\section{DISCUSSÃO}

A União Internacional para a Conservação da Natureza estabelece que uma Área Natural Protegida ou Unidade de Conservação é "uma superfície de terra ou mar consagrada à proteção e manutenção da diversidade biológica, assim como dos recursos naturais e dos recursos culturais associados, e manejada por meios jurídicos e outros instrumentos eficazes", sendo representadas por diferentes categorias (McNEELY et al., 1994). As UC fazem parte do sistema brasileiro de proteção ao meio ambiente e são controladas pelo Instituto Chico Mendes de Conservação da Biodiversidade (ICMBio), órgão federal que compõe o Sistema Nacional de Unidades de Conservação da Natureza (SNUC), instituído, no ano 2.000, pela Lei $\mathrm{N}^{\circ} 9.985$ (ICMBio, 2011).

O município de Uberlândia possui 15 UC legalmente instituídas, assim distribuídas: 10 Parques, três Reservas Particulares do Patrimônio Natural (RPPN) e duas Áreas de Relevante Interesse Ecológico (ARIE). A maioria dessas UC é de domínio municipal e está localizada na zona urbana. Destaca-se o Parque Estadual do Pau Furado, criado em 2007 (Decreto s/ $\mathrm{n}^{\mathrm{o}}$, de 27/01/07), por sua área (cerca de 2.200ha) e pelo fato de estender-se pelos municípios de Araguari e Uberlândia (aproximadamente metade da área em cada município). Excetuando-se ás áreas abrangidas pela Área de Relevante Interesse Ecológico Morada do Sol e pela Área de Relevante Interesse do Lago da Hidrelétrica de Miranda ARIE, as UC de Uberlândia totalizam 1.973,22ha (PMU, 2010; IEF, 2010).

As áreas protegidas dos assentamentos rurais pesquisados equivaleram a $167 \%$ da área total ocupada pelos Parques e Reservas do município de Uberlândia (MG). Ou seja, as APP e as RL dos assentamentos investigados oferecem uma valiosa contribuição para a conservação biológica no município, ao aumentar significativamente $\mathrm{a}$ área destina à proteção ambiental. Convém destacar que algumas dessas áreas apresentam alto grau de degradação ou foram demarcadas em locais originalmente ocupados por pastagens (p.ex. Tangará). No entanto, a partir da sua proteção legal, espera-se que o processo de regeneração natural possa ser restabelecido, recompondo os habitats e promovendo a retomada dos processos ecológicos. Em estudo realizado na Estação Ecológica do Panga, Cardoso et.al. (2009) demonstraram que, em 18 anos, a diminuição das perturbações geradas pelo homem levou a um aumento significativo da densidade arbórea, tanto nas formações savânicas como campestres.

A legislação ambiental brasileira garante a preservação de parte da vegetação das propriedades rurais, sendo que a área de Reserva Legal varia de acordo com o bioma onde cada propriedade está inserida: $80 \%$ da propriedade rural localizada na Amazônia Legal; $35 \%$ da propriedade inserida no bioma Cerrado, nos Estados que compõem a Amazônia Legal; e 20\% nas demais regiões do país (BRASIL, 2001). Entendemos que as RL dos assentamentos rurais pesquisados respeitam a determinação legal, preservando ao menos $20 \%$ da sua área total sob a forma a forma de RL. Os resultados mostram que somente dois assentamentos (Nova Tangará e Rio das Pedras) não atingiram a área esperada, mas essa diferença $(0,25 \%$ em cada assentamento) pode ser atribuída a distorções nas medidas realizadas; ou seja, ao método empregado. Vale lembrar que esses dois assentamentos são os maiores e, portanto, os que apresentam as maiores áreas absolutas de RL.

As áreas das APP são subordinadas às características geomorfológicas e pluviais das regiões nas quais os assentamentos estão implantados e não a sua área total, como acontece com as RL. E é justamente essa diferença que explica a considerável variação observada entre os assentamentos pesquisados, no que diz respeito aos totais de áreas protegidas. Considerando-se que a proporção das RL é praticamente fixa, em torno de $20 \%$, as áreas protegidas refletem não apenas o tamanho dos assentamentos, mas também a variação no tamanho das APP. Desse modo, verificam-se grandes áreas de proteção em grandes assentamentos (p.ex. Nova Tangará e Rio das Pedras), mas também grandes áreas protegidas em assentamentos de dimensões mais modestas, que apresentam grandes áreas de APP, como Canudos e Emiliano Zapata. Note-se que esses dois últimos assentamentos foram os que apresentaram os maiores percentuais de áreas protegidas.

A região do Triângulo apresenta uma grande riqueza de cursos d'água encaixados, além de subsistemas úmidos, compostos de tramas finas e mal delimitadas de caminhos de água em solos saturados, 
Estrutura de Áreas protegidas dos assentamentos de reforma agrária no município de Uberlândia-MG, Brasil:

um estudo de ecologia de paisagem

Paula Guardenho Maywald, Oswaldo Marçal Júnior

nos quais o lençol freático aflora ou está perto de aflorar. São as conhecidas veredas, que têm enorme importância para o bioma Cerrado, por abrigarem nascentes, manterem elevada biodiversidade e serem responsáveis pela regulação de vazão, entre as estações seca e chuvosa. (AB SÁBER, 1971, EMBRAPA, 1975, 1982). Em Uberlândia, onde predomina o relevo dissecado e suavemente plano, é comum o encontro deste tipo de fitofisionomia do Cerrado. Convém notar que a delimitação de APP em áreas de veredas, deve ser feita "em faixa marginal, em projeção horizontal, com largura mínima de cinquenta metros, a partir do limite do espaço brejoso e encharcado" (BRASIL, 2001).

Em condições ideais, cada RL deveria constituir uma única reserva, pois isso evitaria (ou minimizaria) os efeitos negativos da fragmentação de habitat, como a perda de biodiversidade e o aumento do efeito de borda (PRIMACK e RODRIGUES, 2001). Essa situação foi verificada em três assentamentos pesquisados (Nova Palma, Flávia Nunes e José dos Anjos); contudo, a realidade dos demais assentamentos releva uma intensa fragmentação das RL. Isso pode estar relacionado com o próprio processo de escolha dessas áreas.

Segundo Trindade (2004), normalmente, as áreas de vegetação naturais mais preservadas são destinadas às RL, nos projetos de parcelamento dos assentamentos, independentemente da qualidade dos solos, das condições topográficas ou dos princípios ecológicos. Além disso, existem aspectos históricos de ocupação do solo no Brasil Central que não podem ser desconsiderados, sobretudo em termos do processo de desenvolvimento agropecuário e da expansão da fronteira agrícola em áreas de Cerrado (BEZERRA e CLEPS-JUNIOR, 2004). Cada assentamento rural em Uberlândia tem uma história absolutamente particular de ocupação e de demarcação das RL. O assentamento Flávia Nunes, por exemplo, originou-se de uma fazenda que não tinha RL averbada (Fazenda Água Limpa), fato que possibilitou uma seleção mais apropriada para a RL, que foi demarcada em área única, abrigando vegetação característica do Cerrado e em avançado processo de regeneração.

Em Uberlândia são observadas constantes transformações quanto ao uso do solo, computando- -se apenas $17,7 \%$ da área total do município como de vegetação natural ou em regeneração (Cerrado, Campo higrófilo, Mata/ Mata em regeneração), concentrados, principalmente, em áreas onde a utilização para a agricultura é pouco viável economicamente. De modo geral, esta vegetação nativa está restrita às áreas destinadas à proteção dos imóveis rurais, formando pequenos fragmentos em áreas onde o acesso é dificultado pelo relevo (BRITO; PRUDENTE, 2005a; b). Ocorre que fragmentos isolados produzem um efeito negativo sobre a riqueza de espécies, diminuindo a taxa (potencial) de migração ou de recolonização. Dessa forma, a conectividade entre os fragmentos é de grande importância para a dinâmica das populações silvestres, ao garantir o fluxo gênico entre subpopulações (meta populações) (FORMAN, 1983). E entender os padrões espaciais resultantes da fragmentação de habitats se revela de extrema importância para a conservação de populações biológicas (TURNER, 1989; METZGER, 1999).

Dos 30 fragmentos gerados na demarcação das áreas destinadas à RL nos assentamentos rurais de Uberlândia, apenas cinco apresentaram-se completamente isolados. Um fator determinante para essa grande conectividade entre os fragmentos é a sua interconexão com as APP. Tal situação é claramente mostrada nos assentamentos Rio das Pedras e Canudos, nos quais as RL estão muito separadas (cinco e seis fragmentos, respectivamente), mas onde todas foram demarcadas em áreas contíguas às matas ciliares, garantindo a interligação dos fragmentos. Assim, as APP representam não apenas faixas de vegetação de proteção das próprias coleções hídricas, mas se constituem em corredores ecológicos, que possibilitam tanto o fluxo de espécies de interior como de borda (FORMAN, 1983; KAGEYAMA; LEPSCH-CUNHA, 2001; PRIMACK e RODRIGUES, 2001).

De acordo com Forman e Godron (1986), a área do fragmento é uma das principais informações da paisagem, pois nela se baseia o cálculo para os outros índices morfométricos. Vários estudos realizados em ecossistemas florestais fragmentados demonstram uma relação clara entre o tamanho do fragmento e a riqueza e diversidade de espécies (VALENTE e VETTORAZZI, 2002; CORDEIRO, 2002; FLORES-PALÁCIOS; GARCÍA-FRANCO, 2004; BIRELLI e TOREZAN,

Soc. \& Nat., Uberlândia, 25 (1): 75-90, jan/abr/2013 
2007; GUSSON et al, 2009). No presente estudo, os fragmentos apresentaram média de 74,5ha, sendo que mais de $80 \%$ deles mostraram áreas superiores àquelas registradas por Valente e Vettorazzi (2002), em estudo realizado em condições análogas às verificadas em Uberlândia. Convém notar que a área mínima viável do remanescente para a sobrevivência de indivíduos varia de acordo com a espécie focalizada (ALMEIDA; MORO, 2007).

O índice de forma é uma medida que expressa a relação entre a área e o perímetro do fragmento, sendo considerado um parâmetro bastante útil para analisar a vulnerabilidade de fragmentos, especialmente no que diz respeito ao efeito de borda (RANTA et al., 1998). A maioria dos fragmentos de RL pesquisados $(93,5 \%)$ apresentou índice de forma menor do que dois, sendo que oito deles apresentaram índices próximos a um, valor que identifica fragmentos nos quais o efeito de borda é menos intenso (RANTA et al., 1998; PRIMACK; RODRIGUES, 2001). Somente dois assentamentos mostraram fragmentos com índices de forma que podem ser considerados elevados: F2 de Nova Tangará $(\mathrm{IF}=4,08)$ e F1 de Zumbi dos Palmares $(\mathrm{IF}=3,07)$. Note-se que nesses fragmentos, o efeito de borda pode estar sendo minimizado pela grande área ocupada por ambos (992,98ha e 145,88ha, respectivamente). Os altos índices de área interna apresentados por esses dois fragmentos $(91,98 \% \mathrm{e}$ $81,38 \%$, respectivamente) corroboram essa hipótese.

A área interna é máxima em fragmentos grandes e circulares, mas mínima quando o fragmento é pequeno ou sua forma é muito irregular, condição na qual o efeito de borda é especialmente forte (RANTA et al., 1998). Os fragmentos pesquisados apresentaram áreas internas bastante variadas. Um desses fragmentos pesquisados (F2 de Zumbi dos Palmares) não apresentou área interna, em função do seu tamanho extremamente reduzido. Outros dois fragmentos desse mesmo assentamento apresentaram índices de área interna mínimas, o que também pode ser atribuído ao pequeno tamanho dessas áreas ( $0,55 \mathrm{ha} \mathrm{e} 2,07 \mathrm{ha})$. Um desses fragmentos teve duas áreas internas, tamanha foi a sua irregularidade.

O efeito de borda é representado pelas alterações físicas, químicas e biológicas verificadas na área de contato entre um fragmento de vegetação natural e sua matriz de entorno, de natureza antrópica (PRIMACK; RODRIGUES, 2001; LIMA-BARRETO, 2008). Constitui-se na consequência mais significativa da fragmentação (ZHENG; CHEN, 2000), sendo considerada como o principal mecanismo gerador de mudanças na estrutura e dinâmica florestais (NASCIMENTO; LAURENCE, 2006). Embora as bordas sejam componentes comuns de paisagens não perturbadas, proliferam mais rapidamente quando as paisagens são fragmentadas e com maior interferência humana (SISK; HADDAD, 2002). A partir da década de 90, estudos sobre o efeito de borda têm sido desenvolvidos principalmente em biomas florestais onde este efeito geralmente é bem mais evidente do que no Cerrado (CASTRO, 2008). A despeito disso, foram conduzidos diversos estudos enfocando o efeito de borda, tanto na Amazônia e na Mata Atlântica (CASTRO, 2008; PACIÊNCIA; PRADO, 2004; NASCIMENTO; LAURANSE, 2006; LAURANSE et al, 2007), como no Cerrado (CASTRO, 2008; CASTRO; PIVELLO, 2008; LIMA-RIBEIRO, 2008; MENDES; SILVA, 2008; MENDONÇA, 2009).

A maioria dos assentamentos pesquisados tem RL divididas em vários fragmentos, o que pode aumentar a vulnerabilidade dessas áreas. Mas apesar desta fragmentação, cerca de $1 / 3$ dos fragmentos apresentaram IAI superiores a $80 \%$ e cerca de $2 / 3$ mostraram índices maiores do que $50 \%$, o que indica um menor efeito de borda. Isso está associado tanto ao tamanho dos fragmentos quanto à largura de borda considerada no presente estudo $(30 \mathrm{~m})$. Larguras de borda variadas, de até 100 metros, têm sido utilizadas, dependendo de uma série de fatores tais como, o tipo de borda (natural ou artificial), o relevo, o tipo de vegetação do fragmento, a matriz em que este se insere, entre outras características de cada fragmento (PEREIRA, 2003). Neste estudo, optou-se por uma medida mais conservativa $(30 \mathrm{~m})$, com base na largura de borda $(15 \mathrm{~m})$ adotada por LIMA-RIBEIRO (2008), em estudo conduzido em área de Cerrado e com matriz circundante muito semelhante à observada nas áreas pesquisadas. Em que pesem os resultados obtidos, recomenda-se que novas RL sejam demarcadas, sempre que possível, em uma única grande área, a condição mais adequada para minimizar os efeitos de borda (CASTRO, PIVELLO, 2008; MENDES; 
Estrutura de Áreas protegidas dos assentamentos de reforma agrária no município de Uberlândia-MG, Brasil:

um estudo de ecologia de paisagem

Paula Guardenho Maywald, Oswaldo Marçal Júnior

SILVA, 2008; LIMA-RIBEIRO, 2008; LAURANSE et al, 2007; EWERS; DIDHAM, 2008).

Entre os fragmentos analisados, os menores efeitos de borda foram registrados nos assentamentos de Nova Tangará e Rio das Pedras. Neste sentido, esses fragmentos, considerando-se os parâmetros analisados, mostraram ser os mais adequados para cumprir as funções de uma RL, podendo ser tomados como modelos para a definição de novas RL no município. Mas deve ser lembrado que, para que uma população se mantenha viável em um habitat, é necessário considerar não apenas as características estruturais da área em questão, mas também os processos de migrações, colonizações e extinções que ocorrem em todas as populações naturais (PRIMACK e RODRIGUES, 2001). Desta maneira, sugerimos o desenvolvimento de novos estudos nas áreas pesquisadas, abordando outros aspectos ambientais, especialmente a análise de matrizes de entorno, da biogeografia e de metapopulações, de modo a possibilitar uma compreensão mais completa dos padrões espaciais e dos processos ecológicos de áreas fragmentadas.

\section{REFERÊNCIAS}

AB'SABER, A. N. Contribuição à geomorfologia da área dos cerrados. Simpósio sobre o Cerrado. São Paulo: Edusp: 97-103, 1971.

ANDRADE, P. S.; GOMES, J. M. A. Estado e intermediação de interesse: atuação política do segmento agronegócio de grãos na realidade piauiense e a constituição de políticas ambientais. Disponível em: http://www.ufpel.tche.br/isp/ppgcs/eics/dvd/documentos/gts_1lleics/gt7/gt7patricia.pdf. Acesso em 25 mar.2012.

ALMEIDA, C. G.; MORO, R. S. Análise da cobertura florestal do Parque Nacional dos Campos Gerais, Paraná, como subsídio ao seu plano de manejo. Rev. Terr@Plural.1(1): 115-122, Ponta Grossa, 2007.

BASTOS, Yandra Fontes; BRASIL, Ida Cláudia Pessoa. Impacto ambiental, agroecologia e reforma agrária: Fatores que influenciam a transição agroecológica em Áreas de Preservação Permanente (APPs) no projeto demonstrativo do assentamento Jaraguá - Água Boa - MT. ENCONTRO NACIONAL DA ANPPAS, IV. Brasília, DF. 2008. Anais..., Brasília: ANPPAS, 2008.

BATISTTELA, M.; CASTRO, F. de. Institutional design and landscape fragmentation: a comparative study of rural colonization projects in the Brazilian Amazon. US-IALE 2001 ANNUAL SYMPOSIUM, 16. Arizona, 2001. Anais..., Arizona, IALE, 2001.

BEDUCHI FILHO, L.C. Assentamentos rurais e conservação da natureza: Do estranhamento à ação coletiva. São Paulo: Iglu/FAPESP, 2003.

BEZERRA, L. M. C.; CLEPS JR., J. O desenvolvimento agrícola da região Centro-Oeste e as transformações no espaço agrário no estado de Goiás. Caminhos de Geografia, Uberlândia 2(12): 29-49, 2004.

BIRELLI, J.M.; TOREZAN, M.D. A redução da diversidade de epífitas vasculares com a fragmentação florestal é mais intensa para espécies dos estratos inferiores. CONGRESSO DE ECOLOGIA DO BRASIL, VIII. Caxambu, 2007. Anais...., Caxambu: Embrapa, 2007.

BRANDÃO Jr., A.; SOUZA Jr., C. Desmatamento nos Assentamentos de Reforma Agrária na Amazônia. $O$ Estado da Amazônia, 7, 4. Belém, 2006. Disponível em: http:// http://www.imazon.org.br/publicacoes/o-estado-da-amazonia/desmatamento-nos-assentamentos-de-reforma-agraria. Acesso em 10 ago.2008.

BRASIL. Lei $\mathrm{n}^{\circ}$ 4.771, de 15 de setembro de 1965. Institui o novo Código Florestal Brasileiro. Diário Oficial da República Federativa do Brasil. Brasília, 1965.

. Lei $\mathrm{n}^{\circ} 6.938$, de 31 de agosto de 1981. Institui a Política Nacional do Meio Ambiente. Diário Oficial da República Federativa do Brasil. Brasília, 1981.

. Lei $\mathrm{n}^{\circ} 7.803$, de 18 de julho de 1989. Altera a redação da Lei 4.771, de 15 de setembro de 1965. Diário Oficial da República Federativa do Brasil. Brasília, 1989. 
. Medida Provisória $n^{\circ} 2.166-67$, de 23 de agosto de 2001. Altera os parágrafos $1^{\circ}, 4^{\circ}, 14^{\circ}, 16 \mathrm{e}$ 44, e acresce dispositivos à Lei $\mathrm{n}^{\circ} 4.771$, que institui o novo Código Florestal Brasileiro. Diário Oficial da República Federativa do Brasil. Brasília, 2001.

BRITO, J. L. S.; PRUDENTE, T. D Mapeamento do uso da terra e cobertura vegetal do município de Uberlândia-MG, utilizando imagens CCD/CBERS2 . Caminhos da Geografia Uberlândia 13(15): 144-153, 2005a.

. Análise temporal do uso do solo e cobertura vegetal do município de Uberlândia-MG, utilizando imagens ETM+ / Landsat 7 . Sociedade \& Natureza, Uberlândia, 17 (32): 37-46, 2005 b .

CARDOSO, E.; MORENO, M. I. C.; BRUNA, E. M.; VASCONCELOS, H. L. Mudanças fitofisionômicas no Cerrado: 18 anos de sucessão ecológica na Estação Ecológica do Panga, Uberlândia-MG. Caminhos da Geografia. Uberlândia 10(32): 254-268, 2009.

CASTRO, D. M. Efeitos de borda em ecossistemas tropicais: sintese bibliográfica e estudo de caso em fragmentos de cerrado, na região nordeste do estado de São Paulo. São Paulo: USP. Dissertação - Mestrado em Ciências, 2008.

CASTRO, D. M., PIVELLO, V. R. Efeitos de borda cobre o solo em fragmentos de cerradão, na região nordeste do Estado e São Paulo, derivados do manejo agrícola. SIMPÓSIO NACIONAL DO CERRADO, IX; SIMPÓSIO INTERNACIONAL DE SAVANAS TROPICAIS,II, Brasília, 2008. Anais....Brasília: ParlaMundi, 2008.

CORDEIRO, P. H. C. 2002. A fragmentação da Mata Atlântica no sul da Bahia e suas implicações na conservação de psitacídeos, p. 215-227. In: GALETTI M.; PIZO M.A. (Eds). Ecologia e conservação de psitacídeos no Brasil. Belo Horizonte, Melopsittacus Publicações Científicas, 235p.

DATALUTA - Banco de Dados da Luta pela Terra: Relatório 2009 - Minas Gerais. Uberlândia:
LAGEA - Laboratório de Geografia Agrária - IG/ UFU . 2010. Disponível em: http://www.lagea. ig.ufu.br/relatoriosdatalutaminas.html. Acesso em: 06/03/2011.

EMPRESA BRASILEIRA DE PESQUISA AGROPECUÁRIA. Serviço Nacional de Levantamento e Conservação de Solos. Mapa esquemático das regiões Norte, Meio Norte e Centro-Oeste do Brasileira. Rio de Janeiro, 1975. 535p. (Boletim de pesquisa, 17).

. Serviço Nacional de Levantamento e Conservação de Solos. Levantamento de média intensidade dos solos e avaliação de aptidão agrícola das terras do Triângulo Mineiro. Rio de Janeiro 1982, 526p. (Boletim de pesquisa, 1).

EWERS, R. M.; DIDHAM, R.K. Continuous response functions for quantifying the strength of edge effects. Journal of Applied Ecology. London 43: 527-536, 2006.

FERNANDES, E. A. ; CUNHA, N. R.S.; SILVA, R. G. Degradação ambiental no estado de Minas Gerais. Rev. Econ. Sociol. Rural, Brasília 43(1): 179-198, 2005.

FLORES-PALÁCIOS, A.; GARCÍA-FRANCO, J. G. Habitat isolation changes the beta diversity of the vascular epiphyte community in lower montane forest, Veracruz, Mexico. Biodiversity and Conservtion, New York, 17(1): 191-207, 2004.

FORMAN, R. T.T. Corridors in a landscape: their ecological structure and function. Ekologia (CSSR), 2: 375-387, 1983.

FORMAN, R. T.T.; GODRON, Michel. Landscape ecology. New York: Wiley, 1986. 619 p.

GANEM, R. S.; DRUMMOND, J. A.; FRANCO, J. L. A. Análise das áreas prioritárias para a conservação no Bioma Cerrado.SIMPÓSIO NACIONAL DO CERRADO, IX; SIMPÓSIO INTERNACIONAL DE SAVANAS TROPICAIS,II, Brasília, 2008. Anais.... Brasília: ParlaMundi, 2008. 
Estrutura de Áreas protegidas dos assentamentos de reforma agrária no município de Uberlândia-MG, Brasil:

um estudo de ecologia de paisagem

Paula Guardenho Maywald, Oswaldo Marçal Júnior

GIRARDI, E. P. A luta pela terra e sua conquista. Disponível em: http://www4.fct.unesp.br/nera/atlas/ luta_pela_terra.htm Acesso em: 15 jul.2011.

GUSSON, A. E. et al. Características químicas do solo e estrutura de um fragmento de floresta estacional semidecidual em Ipiaçu, Minas Gerais, Brasil. Rodriguésia, Rio de Janeiro 60(2): 403-414, 2009.

INSTITUTO CHICO MENDES. O Instituto. Disponível em: http://www.icmbio.gov.br/quem-somos/o-instituto. Acesso em: 10 dez.2010.

IEF. Áreas Protegidas. Disponível em http://www.ief. mg.gov.br/areas-protegidas. Acesso em: 28 nov.2010.

INCRA Reforma agrária. Disponível em: http:// www.incra.gov.br/portal/index.php?option=com_co ntent\&view $=$ category\&layout $=$ blog\&id=269\&Item $\mathrm{id}=289$ Acesso em: 15 jun.2011.

KAGEYAMA, P.; LEPSCH-CUNHA, N. M. Singularidade da biodiversidade nos trópicos. In: GARAY, I.; DIAS, B. (org.). Conservação da biodiversidade nos trópicos. Petrópolis: Ed. Vozes. p199-214, 2001.

KLINK, C. A.; MACHADO, Ricardo B. A conservação do cerrado brasileiro. Megadiversidade, Belo Horizonte, 1(1):147-155. 2005.

LAURANCE, W. F. et al. Habitat Fragmentation, Variable Edge Effects, and the Landscape-Divergence Hypothesis. Disponível em: http://www.plosone.org/ article/info: doi/10.1371/journal.pone.0001017 Acesso em 16 fev. 2008.

LEITÃO, A. B.; MILLER, J.; AHERN, J.; McGARIGAL, K. Mesuring landscaps: a planner's handbook. USA, Island Press, 2006. 245p.

LIMA-RIBEIRO, Matheus de Souza. Efeitos de borda sobre a vegetação e estruturação populacional em fragmentos de Cerradão, no Sudoeste Goiano, Brasil. Acta. bot. Bras, São Paulo 22(2): 55-545. 2008.
MACHADO, R. B., et al. Estimativas de perda da área do Cerrado brasileiro. Brasília : Conservation International do Brasil, 2004. 26p.

MARGARIDO, T. C. C. MST, degradação ambiental e desaparecimento da fauna. Boletim informativo n.843. Federação da Sagricultura do Estado do Paraná, Curitiba, 2004.

MCGARIGAL, K.; MARKS, B. J. Fragstats version 2.0: espatial pattern analysis program for quantifying landscape structure. Conallis: Oregon state Department, 1994. 67p.

McNEELY, J.A.; HARRISON, J.; DINGWALL, P. (eds.) Protecting Nature: Regional Reviews of Protected Areas. IUCN, Gland, Switzerland and Cambridge, UK. 1994. viii $+402 p$

MENDES, J. D.; SILVA, N. M. Avaliação do efeito de borda em fragmentos do cerrado por meio de métricas de paisagem, no Parque Nacional de Chapada dos Guimarães, Mato Grosso, Brasil.SIMPÓSIO NACIONAL DO CERRADO, IX; SIMPÓSIO INTERNACIONAL DE SAVANAS TROPICAIS,II, Brasília, 2008. Anais....Brasília: ParlaMundi, 2008.

MENDONÇA, A.H. et al. Efeito de borda sobre o estrato arbóreo de cerrado stricto sensu em matriz de pastagem. CONGRESSO DE ECOLOGIA DO BRASIL, IX. São Lourenço, 2009. Anais...., São Lourenço: Embrapa, 2009.

METZGER, J. P. Estrutura da paisagem e fragmentação: Análise bibliográfica. An. Acad. Bras. Ci. Rio de Janeiro. 71(3-1): 445-462, 1999.

. Effects of deforestation pattern and private nature reserves on the forest conservation in settlement areas of the Brazilian Amazon. Revista Biota Neotropica. Disponível em http://eco.ib.usp.br/lepac/ paisagem/Artigos_Jean/Metzger_biota_2001_ deforst.pdf. Acesso em 16 out.2007.

MINISTÉRIO DO MEIO AMBIENTE. Áreas Prioritárias para Conservação, Uso Sustentável e Repar-

Soc. \& Nat., Uberlândia, 25 (1): 75-90, jan/abr/2013 
tição de Benefícios da Biodiversidade Brasileira: Atualização - Portaria MMA $n^{\circ} 9$, de 23 de janeiro de 2007. Brasília: MMA, 2007.

MYERS, N. Et al. Biodiversity hotspots for conservation priorities. Nature, London 403:853-845. 2000.

NASCIMENTO, H. M. N.; LAURANCE, W. F. Efeitos de área de borda sobre a estrutura florestal em fragmentos de floresta de terra firme após 13-17 anos de isolamento. Acta Amazônica. Manaus 36 (2): 183-192, 2006.

PACIÊNCIA, M. L.B.; PRADO, J. Efeitos de borda sobre a comunidade de pteridófitas na Mata Atlântica da região de Una, sul da Bahia, Brasil. Rev. Bras. Bot. São Paulo 27(4): 641-653, 2004.

PEREIRA, J.A.A. Efeitos dos impactos ambientais e da heterogeneidade ambiental sobre a diversidade e estrutura da comunidade arbórea de 20 frgmentos de florestas semidecíduas da região do alto Rio Grande, Minas Gerais. Belo Horizonte: UFMG. Tese - Doutorado em Ecologia, Conservação e Manejo da Vida Silvestre, 2003. 156p.

PEREIRA, M. C.B. Agroecologia: Unindo reforma agrária e conservação ambiental. Revista Brasileira de Agroecologia. Camaragibe 2(2): 1481-1486. 2007.

PREFEITURA MUNICIPAL DE UBERLÂNDIA. Secretaria Municipal de Planejamento e

Desenvolvimento Urbano. Banco de Dados Integrados. Aspectos demográficos. 2009. v.1. Disponível em: http://www.uberlandia.mg.gov. br/?pagina $=$ Conteudo\&id=11. Acesso em: 28/10/2010.

PRIMACK, B. RICHARD. B.; RODRIGUES, EFRAIM. Biologia da Conservação. Londrina. Midiograf, 2001.

RANTA, P. et al. The fragment Atlantic rainforest of Brazil: size, shape end distribution of forests fragments. Biodiversity and Conservation, Verlagshäuser 7: 385-403. 1998.
RODRIGUES, E.R.; MOSCOGLIATO, A. V.; NOGUEIRA, A. C. Viveiros "Agroflorestais" em assentamentos de reforma agrária como instrumentos de recuperação ambiental: um estudo de caso no Pontal do Paranapanema. Cad. biodivers, Curitiba 4(2): 1-8, 2004.

SILVA, R. P. Reforma agrária e sua atualidade. Disponível em: http://www4.fct.unesp.br/nera/boletim. php. Acesso em 05/07/2011.

SISK, T .D.; HADDAD, N. Incorporating the effects of habitat edges into landscape models:effective area models for crossbondary management. In: JINGO, L. \& TAYLOR, W. W. Integrating landscape and ecology into natural resource management. Cambridge, 2002. p. $208-240$

SOARES, J. L. N. Assentamentos de reforma agrária na defesa e conservação do cerrado: $\mathrm{O}$ caso da região Sul do Maranhão. Revista NERA. São Paulo 11(13):144-155. 2008.

TRINDADE, E. S. Avaliação ambiental em áreas de assentamento de reforma agrária: O caso do P.A. Campo Novo, Jequitinhonha, MG. Viçosa: UFV. Tese - Doutorado em Solo e Nutrição de Plantas, 2004. 94p.

TRINDADE JUNIOR, et al. Percepções ao desenvolvimento sustentável: observações para o oeste baiano. SIMPÓSIO REGIONAL DE GEOGRAFIA DO CERRADO, I. Barreiras, 2010. Anais... Barreiras: Siregeo, 2010.

TURNER, M. G. Landscap ecology : the effect of pattern on process. Ann. Rev. Ecol. Syst. Palo Alto 20: 171-197. 1989.

VALENTE, R. O.A., VETTORAZZI, C.A. Avaliação da estrutura florestal na bacia hidrográfica do Rio Corumbataí, SP. Scientia Forestalis, Piracicaba 68: 45-57. 2005.

ZENG, D.; CHEN, J. Edge effects in fragmented landscapes: a generic model for delineating area or edge influences (D-AEI). Ecological Modeling. Maryland 132: 175-190, 2000. 